\title{
Development of a dermal matrix from glycerol preserved allogeneic skin
}

\author{
C. D. Richters $\cdot$ A. Pirayesh $\cdot$ H. Hoeksema $\cdot$ \\ E. W. A. Kamperdijk $\cdot$ R. W. Kreis • \\ R. P. Dutrieux $\cdot$ S. Monstrey $\cdot$ M. J. Hoekstra
}

Received: 1 February 2008/Accepted: 1 May 2008/Published online: 20 May 2008

(C) Springer Science+Business Media B.V. 2008

\begin{abstract}
Dermal substitutes can be used to improve the wound healing of deep burns when placed underneath expanded, thin autologous skin grafts. Such dermal matrix material can be derived from xenogeneic or human tissue. Antigenic structures, such as cells and hairs must be removed to avoid adverse inflammatory response after implantation. In this study, a cost-effective method using low concentrations of $\mathrm{NaOH}$ for the de-cellularization of human donor skin preserved in $85 \%$ glycerol is described. The donor skin was incubated into $\mathrm{NaOH}$ for different time periods; 2, 4, 6 or 8 weeks. These dermal matrix prototypes were analyzed using standard histology techniques. Functional tests were performed in a rat subcutaneous implant model and in a porcine transplantation model; the prototypes
\end{abstract}

C. D. Richters $(\bowtie)$

Euro Skin Bank, P.O. Box 1015, 1940 EA Beverwijk,

The Netherlands

e-mail: nrichters@esb.nl

A. Pirayesh · H. Hoeksema · S. Monstrey

Department of Plastic, Reconstructive \& Aesthetic

Surgery, Burn and Tissue Engineering Centre, University

Hospital, Gent, Belgium

C. D. Richters · E. W. A. Kamperdijk

Department of Molecular Cell Biology \& Immunology, Medical Faculty, Vrije Universiteit (VU) Medical Centre, Amsterdam, The Netherlands

R. W. Kreis · R. P. Dutrieux · M. J. Hoekstra Burns Research Institute, Beverwijk, The Netherlands were placed in full thickness excision wounds covered with autologous skin grafts.

An incubation period of 6 weeks was most optimal, longer periods caused damage to the collagen fibers. Elastin fibers were well preserved. All prototypes showed intact biocompatibility in the rat model by the presence of ingrowing blood vessels and fibroblasts at 4 weeks after implantation. An inflammatory response was observed in the prototypes that were treated for only 2 or 4 weeks with $\mathrm{NaOH}$. The prototypes treated with 6 or 8 weeks $\mathrm{NaOH}$ were capable to reduce wound contraction in the porcine model. In neo-dermis of these wounds, elastin fibers derived from the prototype could be observed at 8 weeks after operation, surrounded by more random orientated collagen fibers. Thus, using this effective low cost method, a dermal matrix can be obtained from human donor skin. Further clinical studies will be performed to test this material for dermal substitution in deep (burn) wounds.

Keywords Skin - Burns - Dermal substitute

\section{Introduction}

Advances in intensive care have resulted in decreased mortality and morbidity, especially with major burns. The current focus in burn care has shifted towards improving the long-term function and appearance of 
the healed skin in conjunction with quality of life. This focus on quality has generated a significant amount of research into the use of skin substitutes to control pain, to enhance wound closure and for a better functional and cosmetic outcome.

The bilayer concept of wound coverage in which both epidermal and dermal analogs are used is gaining widely accepted (Demling et al. 2007). The outer layer of such substitutes has to have a barrier function to protect the wound not only from bacterial contamination, fluid loss, but also, overheating and accumulation of tissue fluids. Dermal elements are important for cell guidance during granulation tissue formation, re-modeling and re-epithelialization. The dermis is essential for restoring normal tissue architecture, diminution of wound contraction and for the prevention of scars.

Wound healing outcome of thin split skin grafted deep burn wounds is often poor, with respect to elasticity and cosmetic appearance due to the lack of a significant dermal component. Paucity of available autograft in major burns necessitates wide mesh grafting which can result in excessive granulation within the interstices and delayed wound healing leading to increased scar formation. Application of a dermal substitute underneath the autologous skin is a possibility to improve the wound healing process (van Zuijlen et al. 2002, Jones et al. 2002).

Dermal substitutes can be derived from xenogenic tissues (mainly pig or bovine) or human skin. In both cases, all cells and hairs have to be removed in such way that the structure of the collagen and elastin fibers is preserved. De-cellularized human donor skin should ideally provide a structurally intact natural three-dimensional collagen and elastin matrix. Human skin can be procured from donors after medical screening and serology tests. Donor skin is available from skin banks. The Euro Skin Bank in Beverwijk, the Netherlands, preserves donor skin in $85 \%$ glycerol. This is a simple, cost-effective method resulting in non-viable but intact skin that can be used as biological dressing on scalds, temporary coverage on excised burns and as a means of wound bed preparation (Mackie et al. 2002). All cells are nonviable but the collagen and elastin fibers are well preserved using this method (Richters et al. 1996). In addition, glycerol $85 \%$ is a slow but effective in-activator of microorganisms (van Baare et al. 1994, 1998a, b).
The method to remove antigenic structures, such as cells from the tissue should be effective without interference with the biocompatibility. Blood vessels and fibroblasts must be able to migrate into the processed tissue after implantation. Ideally, these cells attach to the matrix molecules and will integrate the dermal substitute with the autologous skin placed on it, in this way reducing the contraction of the wound during healing. Within time, the donor collagen will be removed by the cells of the recipient and replaced by new collagen. We hypothesize that the elastin fibers present in donor skin will not be replaced but will serve as "guidance" for the ingrowing blood vessels and fibroblasts. This will results in a more randomly organized new collagen with higher pliability.

$\mathrm{NaOH}$ has been used in the processing of bovine and porcine tissue by Bioplex B.V. (Datascope) to obtain membranes suitable to serve as a template for the formation of neodermis (Hafemann et al. 1999, 2001). This is an effective and low cost method to remove donor cells and hairs. In the present study, we have used a slighty modified method with lower concentrations of $\mathrm{NaOH}$. Human skin was processed for $2,4,6$ or 8 weeks in the $\mathrm{NaOH}$ solution. The obtained prototypes were analyzed using histology and were tested in two different animal models to evaluate their biocompatibility and function as dermal matrix.

\section{Methods}

Preparation of the prototype samples from human skin

Donor skin preserved in $85 \%$ glycerol was obtained from the Euro Skin Bank, Beverwijk, The Netherlands.

After removal of the glycerol by repeated washings in $0.9 \% \mathrm{NaCl}$, the skin was incubated in $0.06 \mathrm{~N}$ $\mathrm{NaOH}$. Incubation was performed for 2, 4, 6, 8 week with agitation, every week the $\mathrm{NaOH}$ solution was replaced. After the treatment with $\mathrm{NaOH}$, neutralization was done using $0.1 \mathrm{~N} \mathrm{HCl}$ for $10 \mathrm{~min}$, followed by washing in $0.9 \% \mathrm{NaCl}$. The obtained materials were preserved into $85 \%$ glycerol until use in the experiments. Before use, the glycerol was removed by incubation in $0.9 \% \mathrm{NaCl}$. 
Samples of the four prototypes $(2,4,6$ or 8 week $\mathrm{NaOH}$ treatment) were taken for histological examination and were tested in two animal models. Subcutaneous implantation to evaluate biocompatibility was performed in a rat model. The different prototypes were also used as a dermal matrix underneath a meshed split skin graft in a porcine full thickness wound model, to test the effects on the outcome of the wound healing process.

\section{Animal models}

\section{Rat implantation model}

Animals Rats were obtained from Harlan CPB (Zeist, The Netherlands). A total of 16 male WAGRij rats (8-10 weeks) were used for the experiments, their weight was 200-220 g at the time of operation. The Animal Welfare Committee of the VU Medical Centre has approved the research protocol. The rats were kept under routine laboratory conditions with free access to water and food. After operation, rats were kept in separate cages.

Experimental procedure Rats were anaesthetized using a mixture of Hypnorm ${ }^{\circledR}$ (fentanyl, Janssen, Berchem, Belgium) and dormicum ${ }^{\circledR}$ (Midazolam, Roche, Mijdrecht, The Netherlands), injected subcutaneously. After shaving, an incision of $1.5 \mathrm{~cm}$ was made up to the subcutaneous fat layer and a pocket of one by $1 \mathrm{~cm}$ was created using scissors. A sample of the prototype of one by $1 \mathrm{~cm}$ was placed in this pocket. The incision was sutured with 4.0 vicryl.

Four groups of rats (four animals per group) were implanted with the 2, 4, 6 or 8 weeks in $\mathrm{NaOH}$ processed prototype. The rats were sacrificed at 4 weeks after operation by $\mathrm{O}_{2} / \mathrm{CO}_{2}$ exposure and after macroscopic inspection; the implants with surrounding tissue were excised and placed in Kryofix solution (ethanol/PEG 400; E. Merck, Darmstadt, Germany). Samples were further processed for histology.

\section{Porcine full thickness wound model}

Animals Four female Yorkshire pigs (weight 30$35 \mathrm{~kg}$ ) were used for the experiments. The Local Animal Welfare committee of the VU Medical Center approved the treatment protocol according to the Dutch law on animal experiments.
Experimental procedure On week before operation, a grid was tattooed by cutting the skin with a scalpel till sub-epidermal depth and applying tattoo ink. Four full thickness excision wounds of $4 \times 4 \mathrm{~cm}$ on each flank were prepared under anesthesia. The prototypes $(2,4,6$ or 8 weeks treated with $\mathrm{NaOH})$ were placed into the different wounds, thus on each pig each prototype was tested $2 \times$. Dermal matrix materials were meshed 1:1.5 for wound drainage only and sutured with a closed mesh pattern into the wound bed. Autologous split skin was recovered from the back of the animal using a dermatome (Aesculap, Germany). The autologous split skin was meshed 1:3 and was sutured with an open mesh pattern on top of the dermal matrix with SurfaSoft (Derma-prof, Moerkapelle, The Netherlands). Wounds were dressed with $0.9 \% \mathrm{NaCl}$ soaked cotton gauzes that were fixed using adhesive bandages (Curafix, Lohmann \& Rausher, Almere, The Netherlands). The dressings were protected from mechanical disturbance using an elastic bandage (Tubigrip, Medeco, Oud-Beijerland, The Netherlands).

The wound dressings were changed on day 4 and 7 after operation, thereafter weekly until all wounds were closed. Surfasoft was removed at day 7 and the take of the autologous skin was scored. Biopsies (4 mm) were taken at day 7, 14, and 21 after wounding. Wound contraction was measured by tracing the edges of the wound and the tattoo grid on transparent film. Contraction was measured using planimetry and expressed as a percentage of the original wound area, corrected for the growth of the animals. Digital photographs were taken for macroscopic wound healing evaluation. Animals were sacrificed at 8 weeks after operation. After macroscopically inspection and planimetry, biopsies covering the full width of the wound were excised, placed in Kryofix and further processed for histology.

\section{Immuno-histochemistry}

Sections of $5 \mu \mathrm{m}$ thickness were cut and stained with the following methods:

- Haematoxilin-Eosin (Gurr, BDH Ltd, Poole, UK), for standard histology.

- Elastica von Giesson (Merck, Darmstadt, Germany), to stain collagen and elastin. 
- $\alpha$-smooth muscle actin ( $\alpha$ SMA) antibody (Sigma) staining. Pericytes, which are present in blood vessels, and myofibroblasts are recognized by this antibody.

After fixation in acetone, slides were incubated with the $\alpha$ SMA antibody for $45 \mathrm{~min}$ at room temperature. Thereafter the slides were washed three times with PBS followed by incubation with a secondary antibody conjugated with horseradishperoxidase (rabbit anti mouse, Dako, Glostrub, Denmark) for $1 \mathrm{~h}$ at room temperature. After washing with PBS, slides were incubated with diaminobenzidine (Dako) to visusalize the positive cells (dark brown color).

Two independent observers analyzed the sections. The outgrowth of the epithelial cells of the autologous split skin graft was scored on the haematoxilineosin (HE) stained sections using a grid with a fixed area in the ocular of the microscope.

The inflammatory response was scored on the HE sections using a grid with in the ocular of the microscope measuring $1 \mathrm{~mm}^{2}$ (10 by 10 fields).

The areas covered with clusters of inflammatory cells (i.e., neutrophilic granulocytes, monocytes and lymphocytes) were counted.

In addition, vascular ingrowth was assessed on the sections stained with the $\alpha$ SMA antibody. The presence of elastin fibers and the orientation of the newly formed collagen fibers were studied on the sections stained with Elastica von Giesson.

\section{Statistical analysis}

The results (outgrowth of epithelial cells and inflammatory response) were analyzed for significance using the non-parametric Kruskal-Wallis test. A $P$-value below 0.05 was considered to be statistically significant.

\section{Results}

Histological examination of the samples

Figure 1 shows the pattern of an intact collagen elastin matrix in a prototype treated for 6 weeks with $\mathrm{NaOH}$. The intact elastin network contains structurally important microfibrils. We observed slight

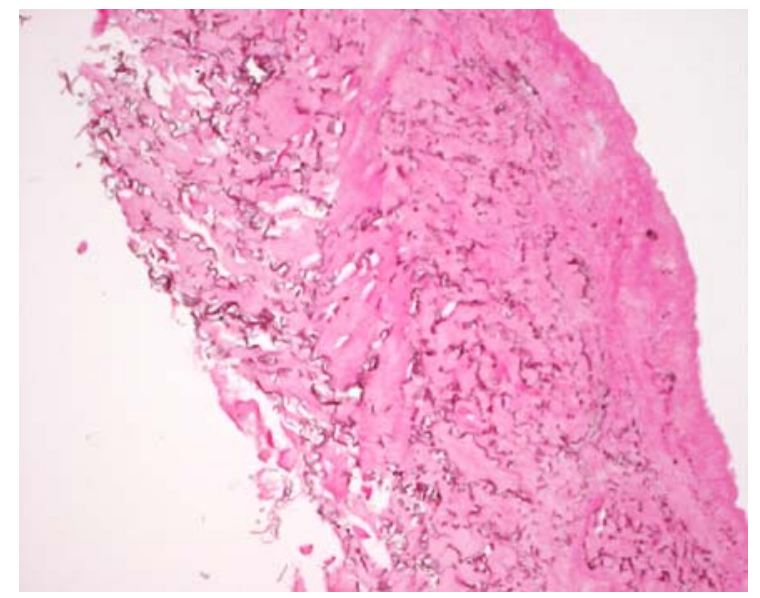

Fig. 1 Section of a prototype dermal substitute treated for 6 weeks with $\mathrm{NaOH}$, stained with Elastica von Giesson staining. Dark fibers are elastin fibers

damage to the collagen in the prototypes that were treated for 8 weeks with $\mathrm{NaOH}$.

Rat implantation model

None of the rats had complications, such as wound infection to the implantation site. The implanted tissues were structurally intact and integrated with the surrounding tissue at 4 weeks after operation.

On sections of the implants, inflammatory cells were observed in the prototypes that were treated with $\mathrm{NaOH}$ for 2 and 4 weeks. This was less pronounced in the prototypes treated for 6 or 8 weeks with $\mathrm{NaOH}$. Fibrovascular ingrowth was present in all implants but more vessels were present in the implants with inflammatory cells (Fig. 2). The implants that were treated with $\mathrm{NaOH}$ for 8 weeks showed more amorphous collagen structure and some damage to the fibers could be observed.

Porcine full thickness wound model

The take of the meshed autologous split skin grafts placed on the different prototypes did not show much difference when scored at day 7 after operation. Between 65 and $80 \%$ (average $75 \%$ ) of the autogolous skin had survived the first days after operation when granulation tissue and new vessels have to grow from the wound bed into the graft.

At day 14 after operation however, clear differences were present between the prototypes. The 


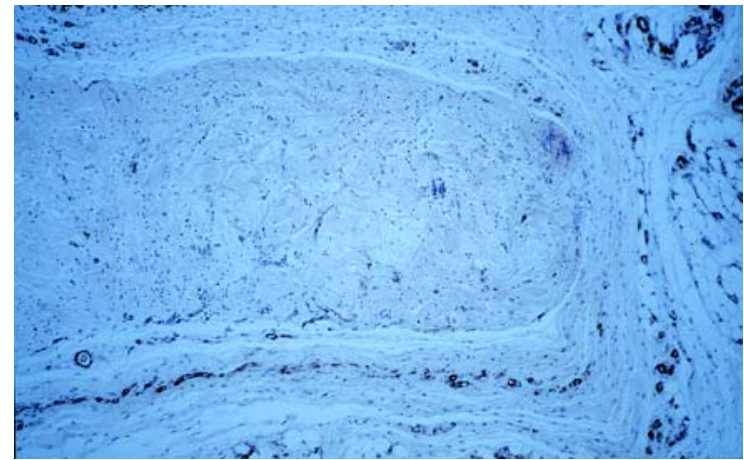

Fig. 2 Representative picture of rat tissue showing an implant, treated 6 weeks with $\mathrm{NaOH}, 4$ weeks after operation. Ingrowth of blood vessels (dark brown stain) and fibroblasts (blue cells) is present

autologous skin grafts placed on the 6 or 8 weeks $\mathrm{NaOH}$ treated prototype has reached $100 \%$ wound closure, whereas on the 2 and 4 weeks $\mathrm{NaOH}$ wounds still showed lesions (Fig. 3). Outgrowth of the epidermis is significant delayed in these latter wounds $(P<0.05$, Kruskal-Wallis test $)$. All wounds were closed at day 21 .

On the sections of biopsies taken at day 14, it appeared that the number of inflammatory cells was significant higher in the prototypes treated for 2 or 4 weeks with $\mathrm{NaOH}$ (Fig. 4, $P<0,05$, Kruskal-Wallis test). This delay in outgrowth of the autologous skin may be caused by this inflammatory response.

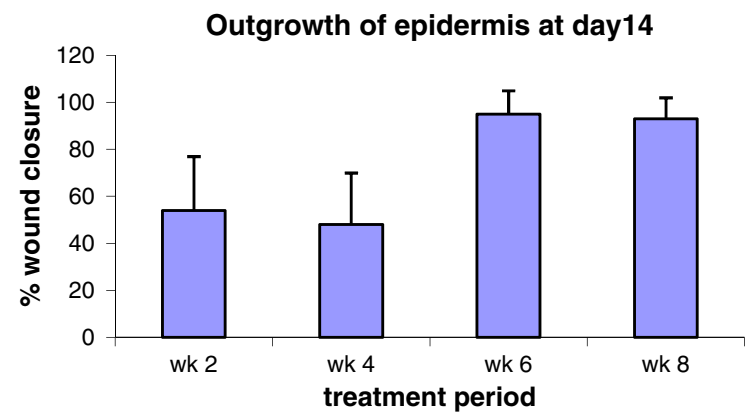

Fig. 3 Porcine excision wounds transplanted with the different prototypes and covered with autologous skin (meshed 1:3). The percentage of the wound bed covered with epithelial cells was counted on biopsies of the wounds at day 7, 14, and 21. At day 14 , outgrowth of the autologous skin is faster on prototypes treated for 6 or 8 weeks with $\mathrm{NaOH}$, resulting in earlier closure of the wound (significant difference in percentage wound closure between wounds treated with the 2 or 4 weeks processed prototype and wounds treated with the 6 or 8 weeks prototype, $P<0.05$, Kruskal-Wallis). Shown are the means ( \pm S.D.) of eight wounds per treatment

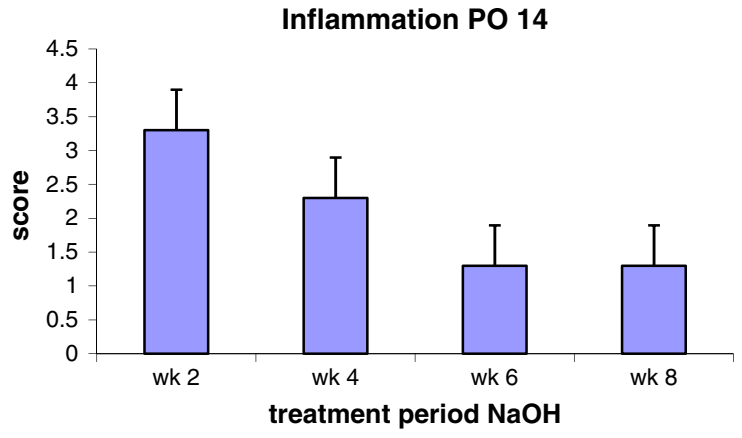

Fig. 4 Longer treatment periods with $\mathrm{NaOH}$ reduced the amount of inflammation in the prototype after implantation under autologous skin grafts in excision wound (porcine model). At day 14 after operation, the number of inflammatory cells was significantly higher in the prototype processed for 2 or 4 weeks compared to the 6 or 8 weeks processed prototypes. Data are expressed as the mean $( \pm$ S.D.) of eight wounds for each treatment

At 8 weeks after operation, wounds transplanted with the 6 or 8 weeks $\mathrm{NaOH}$ treated prototype showed the best results with respect to scar formation and wound contraction. The human derived collagen of the implants could be easily distinguished from newly produced porcine collagen; the implant fibers are thicker and larger.

Most of the collagen of the prototypes was replaced by newly produced collagen and donor derived elastin fibers were still present, especially in the wound treated with the 6 or 8 weeks $\mathrm{NaOH}$ processed prototype. The new collagen fibers showed a more parallel orientation in wounds treated with the 2 or 4 weeks $\mathrm{NaOH}$ processed prototype, whereas in the wounds treated for 6 and 8 weeks randomly organized fibers around the elastic fibers were present (Fig. 5).

\section{Discussion}

The $\mathrm{NaOH}$ de-cellularization method can be an effective method to remove donor cells and hairs from human donor skin. The optimal incubation time with a low concentration of $\mathrm{NaOH}$ is 6 weeks. The prototype treated for 2 or 4 weeks induced an inflammatory infiltrate after implantation and longer periods ( $>8$ weeks) caused clearly visible damage to the extra cellular matrix. The obtained materials still show biocompatibility as demonstrated in the rat 


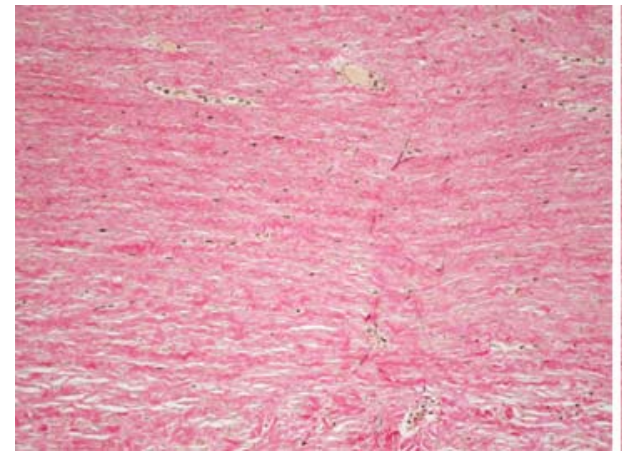

Fig. 5 Sections of the scar tissue, stained with Elastica von Giesson. The sections were prepared from biopsies taken at 8 weeks after operation. The prototypes week 6 (right picture) and week 8 resulted in a scar with more randomly organized

model; by the ingrowth of fibroblasts and blood vessels. The number of vessels was the lowest in the 8 weeks $\mathrm{NaOH}$ prototype, at 4 weeks after implantation. In general, we observed more blood vessel ingrowth in prototypes with higher numbers of inflammatory cells. Experiments using rabbits with AlloDerm (Eppley et al. 2001), an acellular human dermis product of Life Cell Corporation (Branchburg, NJ, USA) placed in subcutaneous pockets showed that within 2 weeks the implant was completely penetrated with blood vessels. Our $\mathrm{NaOH}$ method to obtain acellular human dermis is a low cost method and possibly more stringent compared to AlloDerm, removing more antigenic elements. After processing in $\mathrm{NaOH}$, the dermal matrix tissue can be preserved in $85 \%$ glycerol again until use which makes it easy to store without further damage to the collagen and elastin fibers that might be caused using cyropreservation or freeze-drying techniques.

The results from the porcine excision wound model indicate the 6 or 8 weeks $\mathrm{NaOH}$ treated prototype can serve as a functional dermal substitute; wound contraction was reduced and the newly synthesized collagen bundles showed a more favorable orientation. We observed clear differences between the four prototypes. Treatment with $\mathrm{NaOH}$ for 2 or 4 weeks was apparently too short to remove all antigenic components; an inflammatory response was induced that most probably interfered with the outgrowth of the epidermis from the autologous skin graft. We have observed earlier in other experiments that inflammatory cells can slow down keratinocyte outgrowth; in a rat partial excision wound model

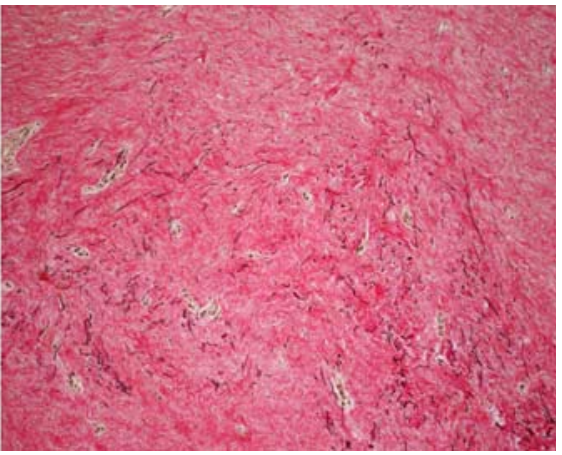

collagen fibers. Elastin fibers are black, most probably originating from the prototypes, surrounded by new porcine collagen. Left picture: biopsy of a wound treated with a prototype that was treated for 2 weeks with $\mathrm{NaOH}$

(Richters et al. 2004) but also in full thickness wound in a porcine model (Richters et al. 2005). These wounds were grafted with the sandwich technique; meshed autologous skin covered with fresh allogeneic skin or glycerol preserved allogeneic skin. The fresh, untreated allogeneic skin induced higher numbers of macrophages and $\mathrm{T}$ cells to the wound bed and the outgrowth of the autologous skin was completed 3 days later compared to wound covered with glycerol preserved allogeneic. The influx of inflammatory cells may have resulted in earlier breakdown of the collagen and elastin in the 2 or 4 weeks $\mathrm{NaOH}$ treated prototypes. Macrophages and neutrophils play an important role in the removal of bacteria and debris in the wound bed. However, their abundant presence in the wound bed may result in damage to the dermal substitute or migrating epithelial cells due to their proteolytic enzymes (Weiss 1989; Ravanti and Kahari 2000). We observed in the sections of the biopsies new collagen produced in an orientation parallel to the epidermis and wound bed suggesting that infiltrating host fibroblasts were not able to use the prototype as scaffold. In the wounds treated with the 6 or 8 weeks $\mathrm{NaOH}$ prototype however, we observed in the sections the fibroblasts could attach to the human tissue derived elastin fibers and synthesize the new fibers around these fibers.

It must be taken into account however that the human derived prototypes are tested in xenogeneic animal models. Walter et al. (1998) have reported two other methods to obtain a-cellular dermal matrix from human or porcine skin using $\mathrm{NaCl}-\mathrm{SDS}$ or dispase-Triton $\mathrm{X}-100$. When tested in a rat wound 
model, the porcine a-cellular dermal tissue showed poor results with respect to graft takes and wound contraction, whereas rat a-cellular dermal tissue was functional (DeSagun et al. 2001; Srivastava et al. 2001). Most likely, there are more differences between rat and porcine tissue compared to human and porcine tissue but we observed also reduced survival of the autologous skin graft on the prototypes that were treated for 2 or 4 weeks with $\mathrm{NaOH}$. In contrast, we show improved results with respect to outgrowth of the epidermis when the prototypes were treated for a longer period (6-8 weeks) with $\mathrm{NaOH}$. Thus, it is possible to evaluate how the method of processing influences the host response after implantation in this model.

Elastin is an extracellular matrix protein that provides elasticity to tissues and organs. It is abundant in organs where elasticity is of major importance, such as blood vessels and skin. The highly specialized elastin matrix in which elastin microfibrils are incorporated and assembled is a key component for skin elasticity; we show that this matrix structure is well preserved using the de-cellularization method with $\mathrm{NaOH}$ on glycerol preserved donor skin. In addition, our results indicate that these donor-derived elastin fibers can be important for the ingrowth of host blood vessels and fibroblasts and in this way will "guide" the fibroblasts in the turn over of donor collagen into host collagen leading to a more natural newly formed dermis.

Thus, in conclusion, using donor skin, a functional dermal substitute based on the natural structure of human skin can be prepared using a cost-effective $\mathrm{NaOH}$ de-cellularization method. We advocate further clinical assessment of this dermal substitute (stored in glycerol, Glyaderm) in combination with autologous skin for effective bilayered skin restoration.

\section{References}

van Baare J, Buitenwerf J, Hoekstra MJ, Du Pont JS (1944) Virucidal effect of glycerol as used in donor skin preservation. Burns 20(Suppl. 1):S77-S80 van Baare J, Ligtvoet EE, Middelkoop E (1998a) Microbiological evaluation of glycerolised cadaveric donor skin. Transplantation 65:966-970

van Baare J, Cameron PU, Vardaxis N, Pagnon J, Reece J, Middelkoop E, Crowe SW (1998b) Comparison of glycerol preservation with cryopreservation methods on HIV1 inactivation. J Burn Care Rehabil 19:494-500

Demling RH, Desanti L, Orgill DP (2007) Biosynthetic skin substitutes: purpose, properties and clinical indications, use of skin substitutes, burnsurgery.org, http://www.burn surgery.org

DeSagun EZ, Botss JL, Srivastava A, Hanumadass M, Walter RJ (2001) Long-term outcome of xenogeneix dermal matrix implantation in immunocompetent rats. J Surg Res 96: 96-106

Eppley BL (2001) Experimental assessment of the revascularization of acellular human dermis for soft tissue augmentation. Plast Recon Sur 107:757-762

Hafemann B, Ensslen S, Erdmann C, Niedballa R, Zuhlke A, Ghofrani K, Kirkpatrick CJ (1999) Use of a collagen/ elastin-membrane for the tissue engineering of dermis. Burns 25:373-384

Hafemann B, Ghofrani K, Gattner HG, Stieve H, Pallua N (2001) Cross-linking by 1-ethyl-3-(3-dimethylaminopropyl)-carbodiimide (EDC) of a collagen/elastin membrane meant to be used as a dermal substitute: effects on physical, biochemical and biological features in vitro. J Mater Sci Mater Med 12(5):437-446

Jones I, Currie L, Martin P (2002) A guide to biological skin substitutes. Br J Plast Surg 55:185-193

Mackie D (2002) Postal survey on the use of glycerol-preserved allografts in clinical practice. Burns 28:S40-S44

Ravanti L, Kähäri VM (2000) Matrix metalloproteinases in wound repair. Int J Mol Med 6:391-407

Richters CD, Hoekstra MJ, Van Baare J, Du Pont JS, Kamperdijk EWA (1996) Morphology of glycerol-preserved human cadaver skin. Burns 22(2):113-116

Richters CD, Du Pont JS, Mayen I, Kamperdijk EWA, Dutrieux RP, Kreis RW, Hoekstra MJ (2004) Effects of a hydrofiber dressing on inflammatory cells in rat partial thickness wound. Wounds 16:63-70

Richters CD, Hoekstra MJ, Du Pont JS, Kreis RW, Kamperdijk EWA (2005) Immunology of skin transplantation. Clin Dermatol 23:338-342

Srivastava A, DeSagun EZ, Jennings LJ, Sethi S, Phuangsab A, Hanumadass M, Reyes HM, Walter RJ (2001) Use of porcine acellular dermal matrix as a dermal substitute in rats. Ann Surg 233:400-408

Walter RJ, Matsuda T, Reyes HM, Walter JM, Hanumadass M (1998) Characterization of acellular dermal matrices (ADMs) prepared by two different methods. Burns 24:104-113

Zuijlen van PP, Angeles AP, Kreis RW, Bos KE, Middelkoop E (2002) Scar assessment tools: implications for current research. Plast Reconstr Surg 109(3):1108-1122

Weiss SJ (1989) Tissue destruction by neutrophils. N Engl J Med 320:365-376 